\title{
Análisis de los efectos sobre el material genético de nutrientes importantes para el crecimiento y desarrollo de la población infantil
}

\section{Gisel Padula, ${ }^{1}$ Rocío Gambaro, ${ }^{2}$ Melisa Mantella ${ }^{3}$ y Analía Seoane 4}

1 IGEVET, Instituto de Genética Veterinaria “Ing. Fernando Noel Dulout” (UNLP-CONICET), Facultad de Ciencias Veterinarias UNLP, 60 y 118 s/n, B-1900-AVW La Plata, Argentina.

Facultad de Ciencias Naturales y Museo UNLP, 60 y 122 s/n, B-190o-AVW La Plata, Argentina.

(iD) https://orcid.org/0000-0001-5390-0043

Correo electrónico: giselpadula@gmail.com

2 IGEVET, Instituto de Genética Veterinaria Ing. Fernando Noel Dulout (UNLP-CONICET), Facultad de Ciencias Veterinarias UNLP, 60 y 118 s/n, B-1900-AVW La Plata, Argentina.

(iD) https://orcid.org/oooo-0001-8376-4740

Correo electrónico: rociogambaro@gmail.com

3 IGEVET, Instituto de Genética Veterinaria Ing. Fernando Noel Dulout (UNLP-CONICET), Facultad de Ciencias Veterinarias UNLP, 60 y 118 s/n, B-1900-AVW La Plata, Argentina.

(iD) https://orcid.org/oooo-0003-3984-3358

Correo electrónico: melimantella@hotmail.com

4 IGEVET, Instituto de Genética Veterinaria Ing. Fernando Noel Dulout (UNLP-CONICET), Facultad de Ciencias Veterinarias UNLP, 60 y 118 s/n, B-190o-AVW La Plata, Argentina.

(iD) https://orcid.org/oooo-0001-9908-538X

Correo electrónico: aseoane@fcv.unlp.edu.ar
Recibido:

3 de marzo de 2020

Aceptado:

31 de julio de 2020

doi: 10.34096/runa.v42i2.7622

\section{Resumen}

Dentro del campo bioantropológico se estudian los cambios somáticos relacionados con el consumo de alimentos y el equilibrio de los nutrientes en el cuerpo. En Argentina, se observa una disminución de desnutrición aguda y un aumento de obesidad, retraso crónico del crecimiento y desnutrición oculta. La desnutrición oculta son conjuntos de carencias específicas de micronutrientes. Una dieta desbalanceada puede incrementar la mutación genética y las aberraciones cromosómicas. Debido a que las deficiencias de micronutrientes no se detectan por antropometría, es importante el diseño de modelos experimentales que permitan investigar sus efectos. El objetivo de este trabajo es presentar los resultados relacionados con la ingesta de macro y micronutrientes de importancia para el normal crecimiento y desarrollo de la población infantil. Se evaluó la inestabilidad genómica asociada a la deficiencia de energía y proteínas, y a las deficiencias y excesos de micronutrientes, y se analizó el rango fisiológico establecido para niños.

\section{Palabras Clave}

Nutrientes; Crecimiento; Población infantil; Modelos experimentales; Estabilidad genómica 
Analysis of the effects on genetic material of nutrients important for the growth and development of the child population

\begin{abstract}
Key words

Nutrition; Growth; Children population; Experimental models; Genomic instabilityy

Within the bioanthropological field somatic changes related to food consumption and the balance of nutrients in the body are studied. In Argentina, a decrease in acute undernutrition and an increase in obesity, chronic growth retardation and hidden hunger are observed. Hidden hunger is a set of specific micronutrient deficiencies. An unbalanced diet can increase genetic mutation and chromosomal aberrations. Because micronutrient deficiencies are not detected by anthropometric techniques, it is important to design experimental models to investigate their effects. The objective of this paper is to present the results related to the intake of macro and micronutrients of importance for the normal growth and development of the child population. Genomic instability associated with energy and protein deficiency, and micronutrient deficiencies and excesses were evaluated and the established physiological range for children was analyzed.
\end{abstract}

\section{Análise dos efeitos no material genético dos nutrientes im- portantes para o crescimento e desenvolvimento da popu- lação infantil}

\section{Resumo}

Palavras-chave

Nutrição; Crescimento; População infantil; Modelos experimentais; Estabilidade genômica
No campo bioantropológico são estudadas mudanças somáticas relacionadas ao consumo de alimentos e ao equilíbrio de nutrientes no organismo. Em Argentina, observamos uma diminuição da desnutrição aguda e um aumento da obesidade, retardo crônico de crescimento e desnutrição oculta. A desnutrição oculta é um conjunto de deficiências específicas de micronutrientes. Uma dieta desequilibrada pode aumentar a mutação genética e as aberrações cromossômicas. Como as deficiências de micronutrientes não são detectadas pelas técnicas antropométricas, é importante projetar modelos experimentais para investigar seus efeitos. O objetivo deste trabalho é apresentar os resultados relacionados à ingestão de macro e micronutrientes de importância para o crescimento e desenvolvimento normal da população infantil. A instabilidade genômica associada à deficiência de energia e proteína e deficiências e excessos de micronutrientes foram avaliadas e o intervalo fisiológico estabelecido para crianças foi analisado.

\section{Nutrientes, crecimiento y desarrollo}

Una nutrición adecuada constituye la base para el bienestar y el desarrollo físico e intelectual de las poblaciones humanas. Se trata de un proceso complejo en el cual intervienen no solo factores económicos sino también culturales, los cuales inciden tanto en los hábitos alimentarios como en los factores sanitarios. En este contexto, desde el campo disciplinar de la antropología biológica existe 
un marcado interés por estudiar los cambios somáticos relacionados con el consumo de alimentos y el equilibrio de los nutrientes en el cuerpo (Díaz, 2011).

La deficiencia asociada de energía, proteínas y micronutrientes lleva a un menor crecimiento somático, en especial en las primeras etapas de la vida en que la velocidad de este es mayor. Esta velocidad puede modularse por el aporte de energía pero la recuperación nutricional es capaz de mejorar solo parcialmente lo perdido y la homeorresis es más eficiente para el peso que para la talla. Los factores genéticos explican la mayor parte de los retardos del crecimiento en los países desarrollados pero en los países en vías de desarrollo la principal influencia corresponde a las deficiencias nutricionales, que pueden generar una malnutrición proteico-energética (MPE) (Waterlow, 1996). Este fenómeno está muy influenciado por otros factores, en particular por las infecciones repetidas (Padula, Salceda, Prozzi, Fernández y Seoane, 2004).

La transición nutricional de nuestro país se caracteriza por la disminución de la malnutrición aguda, un aumento de la obesidad y cifras relativamente altas de retraso crónico del crecimiento y desnutrición oculta (O'Donnell, 1999). La desnutrición oculta ha sido definida como un conjunto de carencias específicas de micronutrientes que se caracterizan por ser altamente prevalentes, de importancia en la salud infantil y que requieren de cierta instrumentación para su diagnóstico (Carmuega, 1999). Este tipo de desnutrición surge como consecuencia de una alimentación inadecuada, no diversificada, marginal con relación a algún nutriente, lo cual determina que las reservas corporales resulten insuficientes para afrontar los esfuerzos del crecimiento, del embarazo, de la lactancia o de una infección (González, Disalvo y Varea, 2006).

Las deficiencias de micronutrientes más importantes y reconocidas en la niñez son las de hierro, yodo, vitamina A, zinc, algunas vitaminas del grupo $\mathrm{B}$ como la riboflavina, el ácido fólico, la niacina y ácidos grasos esenciales. La deficiencia de hierro - una de las principales causas de anemia- afecta el funcionamiento cerebral y el desempeño intelectual del niño, limita de este modo su futura inserción productiva, lo expone a padecer enfermedades más severas y deteriora su salud gestacional y perinatal. La deficiencia de zinc condiciona el crecimiento y el desarrollo, mientras que la falta de vitamina A se relaciona con complejos fenómenos vinculados con la inmunidad y la respuesta a las infecciones.

Las necesidades nutricionales de los niños son más elevadas que las de los adultos; así, las consecuencias de las carencias alimentarias sobre el crecimiento y el desarrollo son también mayores. El individuo adulto requiere de una cantidad de energía y de nutrientes que son necesarios para reemplazar las pérdidas cotidianas o para reponer la energía imprescindible para su metabolismo basal y actividad física. Los niños, en cambio, necesitan una cantidad adicional para satisfacer las demandas del crecimiento. Como consecuencia de la elevada velocidad de crecimiento durante los primeros años de vida, la selección de alimentos con escaso valor o calidad nutricional puede afectar la ingesta de nutrientes críticos y constituir uno de los factores involucrados en el retraso de crecimiento. Este riesgo es aún mayor en niños con bajo o insuficiente peso al nacer y los que padecen una mayor prevalencia de infecciones como consecuencia de un inadecuado saneamiento ambiental o familiar (Carmuega, 1999). Una inadecuada nutrición en la infancia tiene, asimismo, repercusiones sobre las enfermedades de la adultez, el aprendizaje (O'Donnell, 1999) y la salud reproductiva de las mujeres (O'Donnell, Bengoa, Torún, Caballero, Pantín y Peña, 1997). 


\section{Nutrientes y estabilidad genómica}

La exposición in vivo a agentes mutagénicos y carcinogénicos puede incrementar la mutación genética y las aberraciones cromosómicas en las poblaciones humanas (Parry, 1988). Una dieta desbalanceada puede tener efectos similares (Ames, 1998). Muchos minerales y vitaminas actúan como sustrato y/o cofactores en reacciones de mantenimiento del ADN, por lo que su concentración exacta en la célula es crítica. Así, niveles no adecuados de dichas sustancias podrían imposibilitar la actividad de enzimas requeridas para la estabilidad genómica al provocar fracturas de doble o simple cadena sobre el ADN, lesiones oxidativas o ambas (Fenech, 2001 y 2005). Las recomendaciones actuales de ingesta diaria de nutrientes (RDA) (National Research Council [US] Subcommittee on the Tenth Edition of the Recommended Dietary Allowances, 1989) están basadas en la prevención de enfermedades tales como el escorbuto, en el caso de la vitamina $\mathrm{C}$, anemia en el caso del ácido fólico y del hierro, y la pelagra para la niacina. No obstante, los micronutrientes son importantes también en la prevención de enfermedades degenerativas tales como cáncer, enfermedades cardiovasculares, Alzheimer y envejecimiento prematuro (Giovannucci et al., 1998; Morris et al., 1998; Zhang et al., 1998; Ambrosse et al., 1999; Selhub, Bagley, Miller y Rosenberg, 2000; Fenech, 2014). Esto se debe a que estas enfermedades son causadas, en parte, por el daño en el ADN (Holliday, 1995; Ames, 1998, 2001). Dicho daño actuaría como un factor de riesgo para el desarrollo de las enfermedades degenerativas, ya que aumentaría la probabilidad de padecerlas. Hoy en día, la atención está centrada en definir los requerimientos adecuados de minerales y vitaminas para prevenir el daño en el ADN nuclear y mitocondrial. El conocimiento sobre los niveles óptimos de vitaminas y minerales para el mantenimiento de la estabilidad genómica es escaso y desordenado. Sin embargo, existe suficiente evidencia que sugiere que la deficiencia marginal de folato, vitaminas B6, B12, niacina, $\mathrm{C}$ y E, impacta significativamente en el porcentaje de daño genético espontáneo (Fenech, 2014).

Debido a que las deficiencias de micronutrientes requieren de cierta instrumentación para su diagnóstico, y no se detectan por técnicas antropométricas, es de suma importancia el diseño de modelos experimentales que permitan investigar el efecto de estas deficiencias y que aporten información que pueda favorecer el desarrollo de políticas de prevención. Dichas políticas deben hacer hincapié en la importancia de la educación nutricional abocada a la concientización del problema y la generación de una vigilancia social que traspase los marcos de los programas prediseñados.

Para determinar la recomendación diaria dada en la ingesta de un micronutriente puntual es necesario realizar, por un lado, estudios in vitro dosisrespuesta que analicen el efecto de la concentración del micronutriente sobre el daño genético y la citotoxicidad. Y, por el otro, investigaciones in vivo de corte transversal para evaluar la asociación entre la ingesta de nutrientes y los marcadores utilizados.

Si bien los estudios epidemiológicos son eficaces sólo constituyen una parte de las acciones a desarrollar para encarar cuestiones relacionadas con la adaptación de las poblaciones humanas. Brindan una muy valiosa información, ya que son los que mejor nos muestran lo que ocurre en poblaciones reales pero tienen la desventaja de que se necesita una considerable cantidad de tiempo y esfuerzo para llevarlos a cabo. Por su parte, los modelos in vitro son simples, rápidos, confiables y se ha demostrado que son muy eficaces para determinar 
el efecto de los micronutrientes sobre el daño genómico y la citotoxicidad (Kimura, Umegaki, Higuchi, Thomas y Fenech, 2004; Wu, Lyons, Graham y Fenech, 2009; Fenech, 2010). Este modelo es esencial para definir la concentración óptima, el límite superior más seguro de los micronutrientes y el efecto combinado de nutrientes (Fenech, 2010); aunque resulta difícil extrapolar los resultados a lo que sucede in vivo.

Dentro del Laboratorio de Mutagénesis y Carcinogénesis del Instituto de Genética Veterinaria (IGEVET) “Ing. Fernando Noel Dulout” de la Facultad de Ciencias Veterinarias (Universidad Nacional de La Plata-Consejo Nacional de Investigaciones Científicas y Técnicas) surgió una línea de trabajo orientada a la investigación de los efectos sobre el material genético de nutrientes vitales para el mantenimiento de la estabilidad genómica, en niños y adultos. El surgimiento de la línea está en relación con el interés por estudiar la ontogénesis de los individuos, que condujo en primer lugar a la investigación del impacto de la nutrición sobre el crecimiento infantil y, posteriormente, sobre el material genético. Ya que una pérdida de integridad del genoma se asocia de forma prospectiva con un mayor riesgo de enfermedades degenerativas, las cuales pueden afectar el funcionamiento celular y del organismo, y alterar la adaptación del individuo y de las poblaciones humanas. Se trata de una línea de investigación original dentro del campo bioantropológico. En este sentido, varias de las investigaciones que aquí se presentan han sido las primeras en su tipo en llevarse a cabo a nivel nacional, y algunas, a nivel internacional.

El objetivo de este trabajo particular es presentar aquellos resultados relacionados con la ingesta de macro y micronutrientes de importancia para el normal crecimiento y desarrollo de la población infantil. En este sentido, se realizaron investigaciones de carácter epidemiológico e in vitro, en las que se evaluó la inestabilidad genómica asociada tanto a la deficiencia de energía y proteínas como a las deficiencias y excesos de micronutrientes puntuales (zinc, hierro, cobre y vitaminas) y se analizaron las dosis adecuadas de ingesta diaria de estos nutrientes.

Todos los proyectos del grupo de trabajo cuentan con la aprobación del comité de ética pertinente y se desarrollaron conforme con los principios proclamados en la Declaración Universal de los Derechos Humanos de 1948, las normas éticas instituidas por el Código de Nüremberg de 1947 y la declaración de Helsinski de 1964 y sus sucesivas enmiendas y clarificaciones. Se tuvo en cuenta lo normado por la Ley Nacional 25.326 de Protección de Datos Personales, su decreto reglamentario y restantes normas que la complementan. La recolección de datos personales se realizó de acuerdo con el artículo $3^{\circ}$, mediando en todos los casos la autorización escrita en la que figura el consentimiento libre y expreso de los padres o tutores e informado en los términos de los artículos $5^{\circ}$ y $6^{\circ}$. Los padres de los individuos incluidos fueron citados para poder explicarles los objetivos de la investigación y solicitarles su permiso para que sus hijos pudieran participar de ella. Sólo en los casos que aceptaron se les pidió un consentimiento informado confeccionado a tal fin y se procedió al registro de datos y la toma de una muestra de sangre periférica por punción venosa.

\section{Malnutrición proteico-energética primaria, micronutrientes y daño genético}

Algunos autores analizaron los efectos de la MPE primaria sobre el daño genético, tanto en animales de laboratorio como en humanos, y observaron 
resultados contradictorios (Armendares, Salamanca y Frenk, 1971; Thorburn, Hutchinson y Alleyne, 1972; Alu y Murthy, 1993; Terreros, De Luca y Dulout, 1993). Debido a ello, nos propusimos analizar la inducción de aberraciones cromosómicas estructurales (ACE) en una población de niños menores de cinco años con MPE primaria (Padula et al., 2004; Padula, 2008; Padula, Salceda y Seoane, 2009). Se llevaron a cabo estudios de tipo caso-control apareado por sexo y edad en niños malnutridos (25) y eutróficos (25) que concurrieron al Consultorio del Niño Sano del Hospital Interzonal de Agudos y Crónicos Dr. Alejandro Korn (La Plata, Argentina). El protocolo fue aprobado por el comité de ética de dicho hospital. Para determinar el estado nutricional se realizó la evaluación antropométrica de los niños, para lo cual se registraron las variables: peso $(\mathrm{P})$, talla $(\mathrm{T})$, edad $(\mathrm{E})$, perímetro braquial $(\mathrm{PB})$ y los pliegues cutáneos tricipital (PTR) y subescapular (PSE), y se establecieron los indicadores $\mathrm{P} / \mathrm{E}, \mathrm{T} / \mathrm{E}, \mathrm{P} / \mathrm{T}, \mathrm{PB} / \mathrm{E}, \mathrm{PTR} / \mathrm{E}$ y PSE/E, utilizando tanto referencias naciones como internacionales (Sociedad Argentina de Pediatría, 2001; Hamill, Drizd, Johnson, Reed y Roche, 1977). Dicha evaluación fue complementada con el examen clínico realizado por el médico pediatra. Asimismo, se aplicó una encuesta semiestructurada en la que se tuvieron en cuenta: peso al nacimiento, alimentación, enfermedades virales y/o infecciosas y exposición a agentes potencialmente genotóxicos (tales como antibióticos, antiparasitarios, plaguicidas y rayos $\mathrm{X}$ ). Aquellos niños expuestos a rayos $\mathrm{X}$ que hubieran consumido vitaminas o minerales y sufrido alguna infección un mes previo al estudio fueron excluidos. Se realizaron cultivos in vitro de linfocitos y luego se analizaron 100 metafases por cada niño. Se evaluó la frecuencia de ACE total e individual (gaps de mono e isocromátida, fracturas de mono e isocromátida, fragmentos, cromosomas dicéntricos, asociaciones teloméricas y anillos) y se observó que todas las frecuencias (excepto las de anillos, que fueron nulas para ambos grupos) fueron significativamente más altas en los niños con MPE respecto de los niños eutróficos. Este daño en los cromosomas pudo deberse a diversos factores tales como la severa deficiencia de nutrientes esenciales requeridos para la síntesis de $\mathrm{ADN}$ (zinc, hierro y vitamina $\mathrm{A}$ ), el deterioro de los mecanismos de reparación frente a un incremento inusual de aberraciones y/o la ausencia de compuestos protectores frente al daño oxidativo. El hallazgo más importante de este trabajo fue la identificación del daño en los cromosomas como un factor de riesgo para la salud de los niños afectados. Cabe destacar que este estudio, en el que se evaluó la frecuencia de ACE en una población de niños menores de 5 años con MPE primaria, fue el primero de este tipo realizado en Argentina.

Asimismo, analizamos la relación entre la MPE, las infecciones bacterianas y el daño genético (Padula y Seoane, 2008, 2011). En este caso, se obtuvo la frecuencia de ACE en linfocitos de sangre periférica de niños con MPE y eutróficos con y sin infección bacteriana (14 con MPE sin infección, 11 con MPE infectados; 18 eutróficos sin infección, 7 eutróficos infectados). El relevamiento se llevó a cabo en el mismo consultorio mencionado anteriormente. Se realizó la evaluación antropométrica y clínica de los niños y se aplicó una encuesta semiestructurada, de la misma manera que fue explicitado en el trabajo previo. Los niños con anemia o signos evidentes de deficiencia de otros micronutrientes fueron excluidos. Los resultados no arrojaron diferencias significativas en la frecuencia total de ACE dentro del grupo de niños con MPE, independientemente de la presencia de infección. Además, los niños con MPE mostraron una frecuencia total de ACE significativamente mayor a la de los niños eutróficos, infectados o no. Por otra parte, dentro de los eutróficos, aquellos que cursaban infecciones bacterianas tuvieron un aumento significativo de ACE respecto de los sanos. En base a estos hallazgos pudimos concluir que la infección severa se asoció con 
el daño cromosómico, y provocó efectos más notorios en aquellos niños que además estaban malnutridos. Otros autores obtuvieron resultados semejantes (González et al., 2002a y b). Es importante destacar que es difícil discriminar si el daño en el material genético se debió a la malnutrición en sí misma, a las infecciones bacterianas o a todos estos factores actuando sobre los tejidos carentes de nutrientes esenciales. La disminución de energía y aminoácidos esenciales que conlleva la malnutrición puede afectar la síntesis de enzimas requeridas para la reparación del $\mathrm{ADN}$. Como consecuencia, una célula con dificultades para reparar el daño puede mutar más fácilmente y desencadenar procesos de mutagénesis, carcinogénesis, teratogénesis, senescencia temprana o incluso apoptosis (Natarajan, 2002).

Posteriormente se comenzó a investigar el rol de ciertos nutrientes esenciales, tales como el zinc, en la reparación del daño provocado por la malnutrición. Este micronutriente juega un papel vital en el crecimiento longitudinal de los niños y participa en la síntesis y reparación del ADN (Grandy, Weisstaub y López de Romaña, 2010; Sharif, Thomas, Zalewski, Graham y Fenech, 2011). En este sentido, se realizaron una serie de experiencias con el objetivo de analizar el efecto genético de la suplementación in vitro con sulfato de zinc en linfocitos de sangre periférica de niños con MPE sin infecciones asociadas. Se tomaron muestras de 12 niños diagnosticados con MPE en el Observatorio de Calidad de Vida del Hospital Interzonal de Agudos Especializado en Pediatría "Sor María Ludovica" de La Plata. Los cultivos fueron suplementados in vitro con sulfato de zinc (Padula et al., 2014b). La investigación fue aprobada por el Comité Institucional de Revisión de protocolos de Investigación (CIRPI) del mencionado hospital. Se realizó, por un lado, la evaluación antropométrica de los niños (utilizando los indicadores $\mathrm{P} / \mathrm{E}, \mathrm{T} / \mathrm{E}$ y $\mathrm{P} / \mathrm{T}$ con el estándar de la OMS) $\mathrm{y}$, por el otro, se determinó el estado bioquímico de algunos micronutrientes (zinc, folato, vitamina A). Se aplicó una encuesta similar a la utilizada en trabajos anteriores y se tuvieron en cuenta los criterios de exclusión ya especificados. Cada muestra de sangre fue fraccionada y cultivada por separado; una de ellas fue suplementada con sulfato de zinc y la otra no, de este modo, cada niño fue su propio control (figura 1). Los efectos citotóxicos y cromosómicos fueron evaluados a través del ensayo de micronúcleos por bloqueo de la citocinesis (CBMN). A partir del análisis bioquímico se observó que ninguno de los participantes tuvo deficiencia de zinc, folato o vitamina A. Se observó una reducción significativa en las frecuencias de daño cromosómico en los cultivos suplementados respecto de los no suplementados. Asimismo, la división nuclear aumentó de manera significativa en los cultivos suplementados. Comprobamos que tanto los efectos citotóxicos como el daño cromosómico que ocasiona la MPE en los niños pueden ser reparados in vitro con la suplementación de zinc. Debido a que todos los individuos presentaron niveles de zinc dentro del rango fisiológico normal establecido $(80-280 \mu \mathrm{g} / \mathrm{dl})($ Feliu, Piñeiro, López y Slobodianik, 2005), concluimos que dicho rango debería ser revisado para lograr una mejora en los mecanismos de reparación y evitar así la persistencia de daño en el ADN. Este fue el primer trabajo en el cual se evaluó el papel de la suplementación in vitro de sulfato de zinc en sangre periférica de niños con MPE en el mundo.

A partir de estos estudios, surgió el interés por valorar la concentración de ciertos minerales y vitaminas importantes para la población infantil con el propósito de establecer la ingesta adecuada de nutrientes que ayuden a minimizar el daño en el ADN (Padula et al., 2016, 2017; Gambaro, Seoane y Padula, 2018a $\mathrm{y}$ b). Se continuó trabajando con el sulfato de zinc, esta vez con el objetivo de analizar los efectos citostáticos, citotóxicos y genotóxicos provocados por la 
Figura 1. Foto representativa de la suplementación con sulfato de zinc de las muestras de sangre periférica, en cabina de seguridad tipo Il (Área de Cultivo del IGEVET).

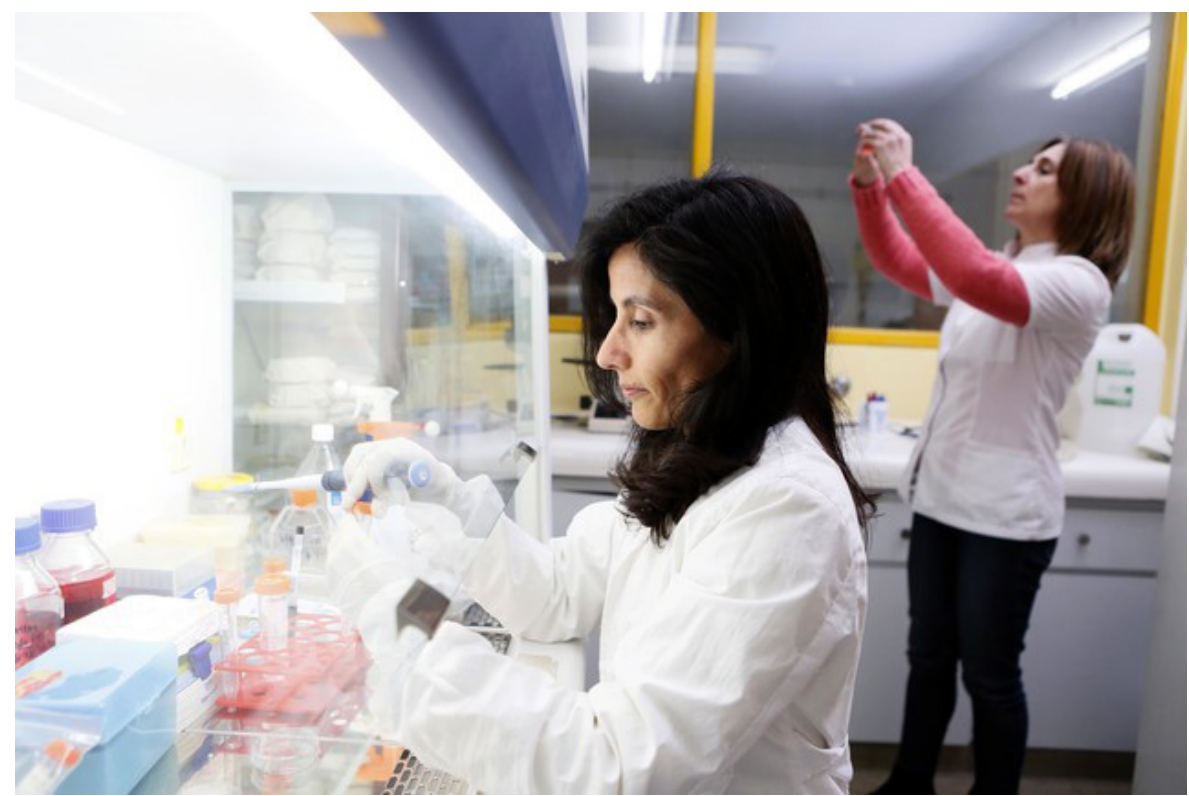

deficiencia y el exceso; asimismo, se evaluó el rango fisiológico normal establecido para niños (Padula, Ponzinibbio y Seoane, 2014a y Padula et al., 2017). El desarrollo experimental se llevó a cabo con muestras de sangre periférica provenientes de seis donantes sanos del mencionado Observatorio de Calidad de Vida y el protocolo fue aprobado por el CIRPI. Se establecieron cultivos deficientes y con exceso de sulfato de zinc y cultivos suplementados con dosis correspondientes al rango fisiológico normal $(12,80,180,280$ y $380 \mu \mathrm{g} / \mathrm{dl})$. Se utilizaron los ensayos CBMN (daño cromosómico, necrosis y apoptosis) y Cometa (daño citomolecular) y se determinó la viabilidad celular con el colorante vital azul de tripano (figura 2). El daño cromosómico y citomolecular resultó significativamente más elevado en los cultivos deficientes $(12 \mu \mathrm{g} / \mathrm{dl})$, en los que fueron suplementados con el límite superior del rango $(280 \mu \mathrm{g} / \mathrm{dl})$ y en aquellos con exceso de sulfato de zinc $(380 \mu \mathrm{g} / \mathrm{dl})$, mientras que la viabilidad fue menor. En los cultivos con exceso se observó además un aumento de la frecuencia de células necróticas, mientras que en los deficientes se observaron las frecuencias más elevadas de células apoptóticas. Otros estudios realizados in vitro también mostraron altos niveles de daño y falla en los mecanismos de reparación del ADN en células deficientes en zinc (Dreosti, 2001; Ho y Ames, 2002; Yan, Song, Wong, Hardin, y Ho, 2008; Sharif, Thomas, Zalewskib y Fenech 2012) y aquellas con exceso (Sharif et al., 2011, 2012). En nuestro trabajo, las dosis más beneficiosas fueron las del límite inferior y medio del rango fisiológico normal $(80$ y $180 \mu \mathrm{g} / \mathrm{dl})$. Estos resultados obtenidos in vitro permitieron sugerir que la suplementación con dosis intermedias de sulfato de zinc $(80$ y 180 g/dl) sería beneficiosa para la salud por su contribución al mantenimiento de la estabilidad genómica, mientras que la suplementación con la concentración correspondiente al límite superior del rango fisiológico normal establecido para niños incrementaría la inestabilidad, tanto como lo hacen la deficiencia y el exceso. Estos hallazgos son importantes, ya que para prevenir el daño en el ADN es necesario definir los niveles adecuados de ingesta de minerales, con el propósito de establecer recomendaciones que contemplen la prevención del desarrollo de enfermedades. Se trata del primer estudio en el que se analizan los efectos de la deficiencia y el exceso de zinc, así como el rango fisiológico normal establecido para niños, en la Argentina. 


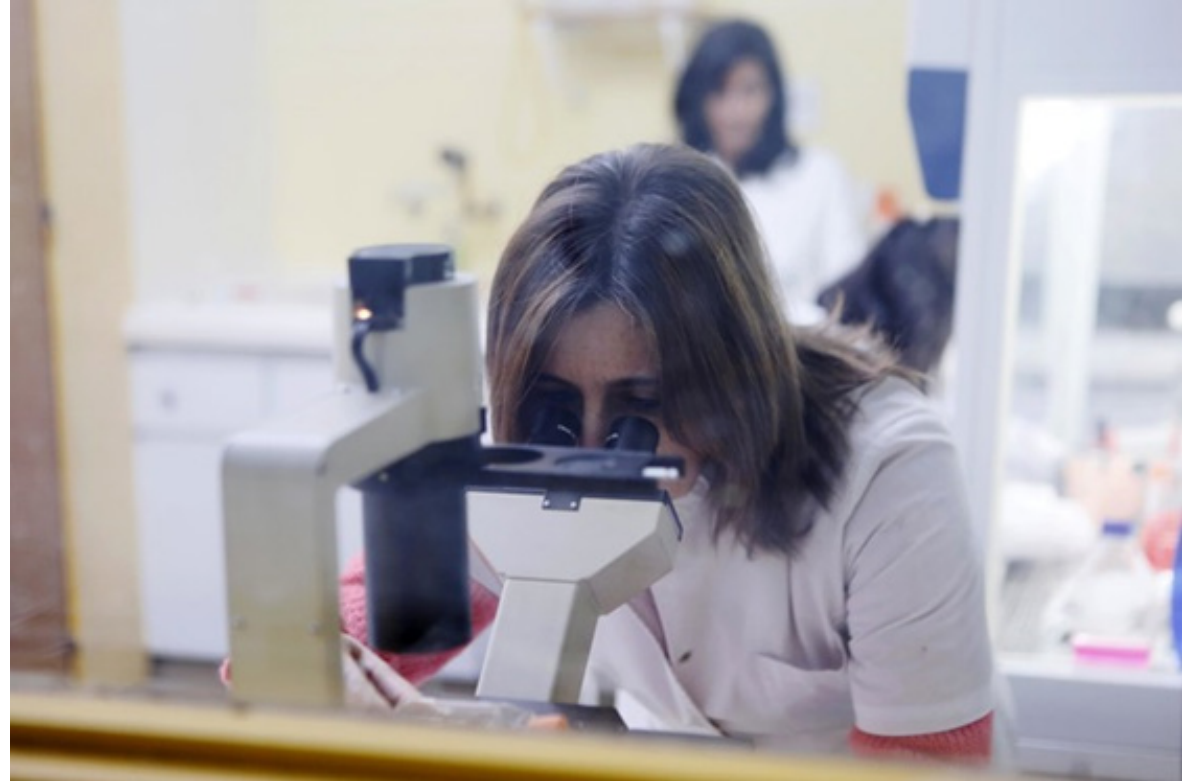

Por su parte, estudiamos los efectos sobre el material genético de dos tipo de suplementación preventiva de la anemia por deficiencia de hierro (ADH) con sulfato ferroso. La deficiencia de hierro es la carencia nutricional más prevalente y la principal causa de anemia a escala mundial. Los grupos poblacionales con mayor riesgo son los niños de entre 6 y 24 meses de edad y las mujeres embarazadas (Gambaro et al., 2018a y b). En los niños la causa más común es el aumento del requerimiento ferroso relacionado con la mayor velocidad de crecimiento. La Sociedad Argentina de Pediatría recomienda como tratamiento preventivo de la anemia la suplementación diaria con sulfato ferroso (Comité Nacional de Hematología, Oncología y Medicina Transfusional y Comité Nacional de Nutrición, 2017). Sin embargo, a partir de la década del noventa algunos autores plantearon como tratamiento alternativo la suplementación con una dosis semanal única, la cual provocaría menos efectos adversos (Tee et al., 1999; Viteri, Ali y Tujague, 1999; Agarwal, Gomber, Bisht y Som, 2003; Zalles Cueto, Rojas Meneces, Rojas Soto y Sejas, 2005; Peña Rosas, De Regil, Gomez Malave, Flores Urrutia y Dowswell, 2015). El hierro participa en las reacciones de óxido-reducción de la célula y en estas se generan especies reactivas del oxígeno (ROS) las cuales pueden ocasionar daño permanente en las proteínas, en los lípidos y en el ADN (Aksu et al., 2010; Jomova y Valko, 2011; Lin, Li, Jia, Ward y Kaplan, 2011; Prá, Franke, Henriques y Fenech, 2012). En trabajos donde se evaluó el efecto del tratamiento terapéutico de la $\mathrm{ADH}$ con sulfato ferroso se observó un aumento de fracturas de $\mathrm{ADN}$, micronúcleos y bases oxidadas en niños y en animales de laboratorio (Aksu et al., 2010; Prá et al., 2008, 2012). Nos propusimos entonces analizar el daño genómico inducido por ambos tipos de suplementación preventiva de la ADH en linfocitos humanos cultivados in vitro (Gambaro et al., 2018 a y b). Se utilizaron muestras de 10 donantes sanos que concurrieron al Observatorio de Calidad de Vida; el protocolo fue aprobado por el Comité Consultivo de Bioética de la Universidad Nacional de La Plata. Los cultivos fueron suplementados in vitro por 7 días con dosis semanales y diarias de sulfato ferroso (figura 3); la concentración de hierro se midió a través de espectrometría por absorción atómica. Se evaluó la viabilidad celular mediante el ensayo de una sal de tetrazolio mejor conocida como MTT (actividad mitocondrial) y el ensayo de rojo neutro (actividad lisosomal).
Figura 2. Foto representativa de la determinación de la viabilidad celular con el colorante vital azul de tripano por microscopía óptica (Área de Cultivo del IGEVET) 
Figura 3. Foto representativa de la suplementación con sulfato de hierro de las muestras de sangre periférica, en cabina de seguridad tipo II (Área de Cultivo del IGEVET).

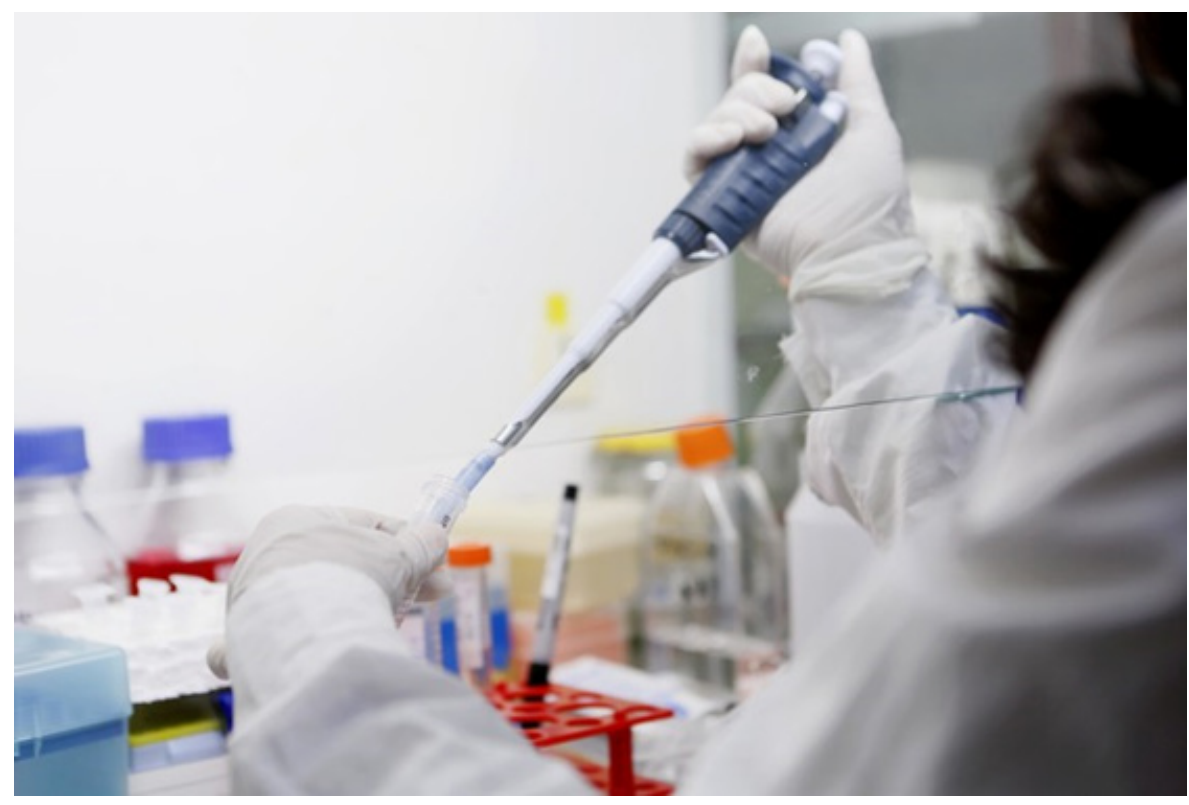

Se analizó la peroxidación lipídica por medio de la técnica de T-Bars y se midió la respuesta antioxidante con la determinación de las enzimas superóxido dismutasa SOD y catalasa. Por último, se evaluaron los efectos sobre los cromosomas con el ensayo CBMN y sobre el ADN con el ensayo Cometa. Todos los biomarcadores analizados mostraron una disminución del daño, del estrés oxidativo y un aumento de la viabilidad durante la suplementación semanal, lo que sugiere que una menor producción de radicales libres del oxígeno disminuye el estrés oxidativo y, como consecuencia, el daño genético. La evidencia en la literatura mostró que el tratamiento terapéutico con sulfato ferroso de la ADH causó un mayor daño en el ADN (Prá et al., 2008). Mientras que en otros trabajos se observó que el estrés oxidativo y la respuesta antioxidante no presentaron incrementos (Hacıhamdioglu et al., 2013; Zaka-Ur-Rab, Adnan, Ahmad e Islam, 2016). Los resultados obtenidos permiten proponer a la suplementación semanal como una mejor alternativa para la prevención de la anemia ferropénica. Cabe destacar que este fue el primer estudio a nivel mundial donde se realizó la comparación de ambos tipos de suplementación preventiva (diaria y semanal) y se evaluó el estrés oxidativo, la respuesta antioxidante y el daño en el ADN.

Actualmente, se están investigando los efectos de la suplementación combinada de algunos minerales y vitaminas. En un caso, estamos comprobando los efectos de la suplementación conjunta de hierro con vitaminas E o C; y en otro caso, se está evaluando el efecto combinado del cobre y el zinc. Ambos estudios se encuentran en curso.

\section{A modo de cierre}

Todos los nutrientes mencionados juegan un rol fundamental no sólo en el crecimiento y desarrollo de los niños sino en el mantenimiento de la estabilidad genómica y el desarrollo de enfermedades degenerativas. En este sentido, la prevención del daño en el ADN es un parámetro importante para la definición de nuevos valores de referencia dietéticos, porque se ha demostrado repetidamente que una pérdida de integridad del genoma se asocia de forma prospectiva con un mayor riesgo de enfermedades degenerativas. De este modo, 
es importante diseñar protocolos de investigación que permitan, por un lado, conocer la situación nutricional de los niños para contribuir a la reformulación de estrategias de atención primaria de la salud infantil teniendo en cuenta el criterio de factor de riesgo para el desarrollo de enfermedades degenerativas. Y, por el otro, definir los valores adecuados de ingesta diaria de nutrientes de manera individual o combinados. Las actuales recomendaciones de ingesta proporcionan una guía para la prevención de enfermedades causadas por deficiencia o exceso de ciertos nutrientes. Determinar estos extremos es importante, pero el mayor desafío será establecer la ingesta adecuada de micronutrientes a partir de mejores dietas, fortificación de alimentos y/o suplementación farmacológica, con el propósito de optimizar el funcionamiento celular y del organismo, para favorecer la adaptación del individuo y de las poblaciones humanas. Esto se vuelve especialmente importante en la infancia, ya que los requerimientos nutricionales únicos de los niños los hacen inusualmente susceptibles a una ingesta inadecuada de nutrientes. Definir la ingesta adecuada de nutrientes podría ser el mejor camino para minimizar el daño en el ADN con el fin de mejorar la salud y prolongar la esperanza de vida.

\section{Financiamiento}

Este trabajo fue financiado a través de dos subsidios, uno otorgado por el CONICET (Proyectos de Investigación Plurianual Nro. 0657) y otro por la UNLP (Programa de Incentivos V246).

\section{Agradecimientos}

Los autores agradecen tanto al CONICET como a la UNLP por el financiamiento recibido. Asimismo, agradecemos la colaboración del Técnico César Bianchi.

\section{Biografía}

Gisel Padula: Lic. en Antropología. Dra. en Cs. Naturales. Investigadora Adjunta CONICET. Profesora Adjunta Facultad de Cs. Naturales y Museo (UNLP).

Rocío Gambaro: Lic. en Antropología. Dra. en Cs. Naturales. Becaria Posdoctoral UNLP. Melisa Mantella: Lic. en Antropología. Colaboradora Instituto de Genética Veterinaria (IGEVET).

Analía Seoane: Lic. en Biología. Dra. en Cs. Naturales. Investigadora Independiente CONICET. Profesora Adjunta Facultad de Cs. Veterinarias, UNLP. 


\section{Q Referencias bibliográficas}

" Agarwal, K. N., Gomber, S., Bisht, H. y Som, M. (2003). Anemia prophylaxis in adolescent school girls by weekly or daily iron-folate supplementation. Indian Pediatric, 40, 296-301.

» Aksu, B. Y., Hasbal, C., Himmetoglu, S., Dincer, Y., Koc, E. E., Hatipoglu, S. y Akcay T. (2010). Leukocyte DNA damage in children with iron deficiency anemia: effect of iron supplementation. European Journal of Pediatrics, 69, 951-956. doi: 10.1007/so0431-010-1147-1

» Alu, V.y Murthy, P. B. (1993). Chromosomal abnormalities in starved and marginally malnourished rats and in utero upon rehabilitation. Experientia, 49, 258-262. doi: 10.1007/ BFo1923535

» Ambrosse, C. B., Freudenheim, J. L., Thompson, P. A., Bowman, E., Vena, J. E., Marshall, J. R., Laughlin, R., Nemoto, T., Shields, P. G. y Graham, S. (1999). Manganese superoxide dismutase genetic polymorphisms, dietary antioxidants and risk of breast cancer. Cancer Research, 59(3), 602-606.

"Ames, B. N. (1998). Micronutrients prevent cancer and delay ageing. Toxicology Letters, 102/103, 5-18. doi: 10.1016/s0378-4274(98)00269-o

"Ames, B. N. (2001). DNA damage from micronutrient deficiencies is likely to be a major cause of cancer. Mutation Research, 475, 7-20. doi: 10.1016/s0027-5107(01)0o070-7

" Armendares, S., Salamanca, F. y Frenk, S. (1971). Chromosome abnormalities in severe protein calorie malnutrition. Nature, 232, 271-273. doi: 10.1038/232271ao

"Carmuega, E. (1999). La calidad de la dieta de los dos primeros años de vida. En A. O’Donnell y E. Carmuega (Eds.). Hoy y Mañana. Salud y calidad de vida de la Niñez Argentina (157-194). Buenos Aires: Centro de Estudios sobre Nutrición Infantil (CESNI).

»Comité Nacional de Hematología, Oncología y Medicina Transfusional y Comité Nacional de Nutrición (2017). Deficiencia de hierro y anemia ferropénica. Guía para su prevención, diagnóstico y tratamiento. Archivos Argentinos de Pediatría, 115(4), s68-s82/s68. doi: http://dx.doi.org/10.5546/aap.2017.568

» Díaz, M. E. (2011). ¿Antropología de la alimentación o antropología de la Nutrición? CATAURO Revista Cubana de Antropología, 12(24), 13-28.

"Dreosti, I. E. (2001). Zinc and the gene. Mutation Research, 475, 161-167. doi: 10.1016/ s0027-5107(01)00067-7

» Feliu, S. M., Piñeiro, A., López, C. y Slobodianik, N. H. (2005). Valores de referencia de cobre, zinc y selenio en niños. Acta Bioquímica Clínica Latinoamericana, 39, 459-462.

"Fenech, M. (2001). Recommended dietary allowances (RDAs) for genomic stability. Mutation Research, 480-481, 51-54. doi: 10.1016/soo27-5107(01)00168-3

"Fenech, M. (2005). The genome health clinic and genome health nutrigenomics concepts: diagnosis and nutritional treatment of genome and epigenome damage on an individual basis. Mutagenesis, 20(4), 255-269. doi: 10.1093/mutage/geio40

»Fenech, M. F. (2010). Dietary reference values of individual micronutrients and nutriomes for genome damage prevention: current status and a road map to the future. American Journal of Clinical Nutrition, 91, 1438S. doi: 10.3945/ajcn.2010.28674D

"Fenech, M. (2014). Nutriomes and Personalised Nutrition for DNA Damage Prevention, Telomere Integrity Maintenance and Cancer Growth Control. Cancer Treatment Research, 159, 427-441. doi: 10.1007/978-3-642-38007-5_24 
» Gambaro, R. C., Seoane, A. I. y Padula, G. (2018a). Comparación in vitro de la frecuencia de micronúcleos provocada por dos formas de administración del tratamiento preventivo de la anemia ferropénica. Revista Argentina de Antropología Biológica, 20(1), 1-9. doi: https://doi.org/10.17139/raab.2018.0020.01.02

» Gambaro, R. C., Seoane, A. I. y Padula, G. (2018b). Oxidative Stress and Genomic Damage Induced In Vitro in Human Peripheral Blood by Two Preventive Treatments of Iron Deficiency Anemia. Biological Trace Element Research, 190, 318-326. doi: https://doi. org/10.1007/s12011-018-1576-7

» Giovannucci, G., Stampfer, M. J., Colditz, G. A., Hunter, D. J., Fuchs, C., Rosner, B. A., Speizer, F. E. y Willett, W. C. (1998). Multivitamin use, folate and colon cancer in women in the Nurses' Health Study. Annals of Internal Medicine, 129, 517-524. doi: 10.7326/00034819-129-7-199810010-00002

» González, C., Najera, O., Cortés, E., Toledo, G., López, L., Betancourt, M. y Ortíz, R. (2002a). Hydrogen peroxide-induced DNA damage and DNA repair in lymphocytes from malnourished children. Environmental and Molecular Mutagenenesis, 39(1), 33-42. doi: 10.1002/em.10038

» González, C., Najera, O., Cortes, E., Toledo, G., Lopez, L., Betancourt, M. y Ortiz, R. (2002b). Susceptibility to DNA damage induced by antibiotics in lymphocytes from malnourished children. Teratogenesis, Carcinogenesis and Mutagenenesis, 22(2), 147-158.

» González, H., Disalvo, L. y Varea, A. (2006). Repercusión de las deficiencias de micronutrientes en salud pública. Ludovica Pediátrica, 7(1), 10-15.

» Grandy, G., Weisstaub, G. y López de Romaña, D. (2010). Deficiencia de hierro y zinc en niños. Revista de la Sociedad Boliviana de Pediatría, 49(1), 25-31.

»Hacıhamdioglu, D. Ö., Kurekci, A. E., Gursel, O., Atay, A. A., Balamtekin, N., Aydın, A., Ha imi, A. y Ozcan, O. (2013). Evaluation of lipid peroxidation and antioxidant system in healthy iron-replete infants receiving iron prophylaxis. Nutrition, 29, 138-142. doi: 10.1016/j.nut.2012.05.009

» Hamill, P. V., Drizd, T. A., Johnson, C. L., Reed, R. B. y Roche, A. F. (1977). NCHS growth curves for children birth-18 years. United States. Vital Health Stat, 11(65), i-74.

» Ho, E. y Ames, B. N. (2002). Low intracellular zinc induces oxidative DNA damage, disrupts $p_{53}$ NFkappa $B$, and $A_{1}$ DNA binding, and affects DNA repair in a rat glioma cell line. Proceedings of the National Academy of Sciences of the United States of America, 99, 16770-16775. doi: 10.1073/pnas.222679399

» Holliday, R. (1995). Understanding ageing. Cambridge (RU): Cambridge University Press.

» Jomova, K. y Valko, M. (2011). Importance of iron chelation in free radical-induced oxidative stress and human disease. Current Pharmaceutical Design, 17(31), 3460-3473. doi: $10.2174 / 138161211798072463$

» Kimura, M., Umegaki, K., Higuchi, M., Thomas, P. y Fenech, M. (2004). Methylenetetrahydrofolate reductase $\mathrm{C} 677 \mathrm{~T}$ polymorphism, folic acid and riboflavin are important determinants of genome stability in cultured human lymphocytes. Journal of Nutrition, 134, 48. doi: 10.1093/jn/134.1.48

» Lin, H., Li, L., Jia, X., Ward, D. M. y Kaplan, J. (2011). Genetic and Biochemical Analysis of High Iron Toxicity in Yeast. The Journal of Biological Chemestry, 286(5), 3851-3862. doi: 10.1074/jbc.M110.190959

» Morris, M. C., Beckett, L. A., Scherr, P. A., Herbert, L. E., Bennett, D. A., Field, T. S. y Evans, D. A. (1998). Vitamin E and Vitamin C supplement use and risk of incident Alzheimer disease. Alzheimer Disease \& Associated Disorders, 12(3), 121-126. doi: 10.1097/00002093199809000-00001 
» Natarajan, A. T. (2002). Chromosome aberrations: past, present and future. Mutaion. Research, 504, 3-16. doi: 10.1016/s0027-5107(02)00075-1

» National Research Council (US) Subcommittee on the Tenth Edition of the Recommended Dietary Allowances (1989). Recommended Dietary Allowances, 10.ma edición. Washington (DC): National Academies Press.

» O’Donnell, A. (1999). Una visión de la problemática nutricional de los niños argentinos. En A. O’Donnell y E. Carmuega (Eds.). Hoy y Mañana. Salud y calidad de vida de la Niñez Argentina (119-156). Buenos Aires: CESNI.

» O’Donnell, A., Bengoa J. M., Torún, B., Caballero, B., Lara Pantín, E., Peña, M. (Eds.) (1997). Nutrición y alimentación del niño en los primeros años de vida. Washington DC: Organización Panamericana de la Salud, OPS/OMS.

»Padula, G. (2008). Malnutrición infantil. Evaluación antropométrica y consecuencias cromosómicas. La Plata: Ediciones al Margen.

»Padula, G. y Seoane, A. I (2008). Chromosomes effects of infections in malnourished and euthrophic childrens of Gran La Plata. Journal of Basic \& Applied Genetics, 19(1), 15-20.

»Padula, G. y Seoane, A. I. (2011). Genotoxic effects of Malnutrition and Infections in Argentinean children. En J. B. Knudsen (Ed.). Malnutrition: Risk Factors, Health Effects and Prevention (1-10). Nueva York: Nova Science Publishers.

»Padula, G, Salceda S. y Seoane, A. I. (2009). Protein-energy malnutrition contributes to increase structural chromosomal alterations frequencies in Argentinean children: a case-control study. Nutrition Research, 29(1), 35-40. doi: 10.1016/j.nutres.2008.09.013

» Padula, G., Ponzinibbio, M. V. y Seoane, A. I. (2014a). Suplementación in vitro con sulfato de zinc de cultivos de sangre periférica: inestabilidad genómica asociada a la deficiencia y al exceso. Journal of Basic \& Applied Genetic, 25(2), 5-16.

»Padula, G., González, H., Varea, A. y Seoane, A. (2014b). Protein Energy-Malnutrition: Does the in vitro zinc sulfate supplementation improve chromosomal damage repair? Biological Trace Element Research, 162(1-3), 64-71. doi: 10.1007/s12011-014-0109-2

»Padula, G., Ponzinibbio, M. V. y Seoane, A. (2016). Impact of folic acid supplementation on low dose ionizing radiation-induced genomic instability in vitro. Indian Journal of Experimental Biology, 54, 537-543.

»Padula, G., Ponzinibbio, M. V, Gambaro, R. y Seoane, A. (2017). Genomic instability related to zinc deficiency and excess in an in vitro model: Is the upper estimate of the physiological requirements recommended for children safe? In Vitro Cellular \& Developmental Biology - Animal, 53, 586-592. doi: 10.1007/s11626-017-0146-1

»Padula, G., Salceda, S., Prozzi, M., Fernandez, A. y Seoane, A. (2004). Alteraciones cromosómicas en niños con Malnutrición Proteico-Energética: resultados preliminares. Ludovica Pediátrica, 6(2), 43-50.

» Parry, J. M. (1988). Studies upon the genetic effects of environmental chemicals: the coordinated research programme of the European Economic Community. Mutagénesis, 3(2), 105-136. doi: 10.1093/mutage/3.2.105

» Peña-Rosas, J. P., De-Regil, L. M., Gomez Malave, H., Flores-Urrutia, M. C., y Dowswell, T. (2015). Intermittent oral iron supplementation during pregnancy. The Cochrane database of systematic reviews, 2015(10), CDoo9997. doi:10.1002/14651858.CDoo9997.pub2

» Prá, D., Franke, S. I. R., Giulian, R., Yoneama, M. L., Dias, J. F., Erdtmann, B. y Henriques, J. A. P. (2008). Genotoxicity and mutagenicity of iron and copper in mice. Biometals, 21(3), 289-297. doi: 10.1007/s10534-007-9118-3 
»Prá, D., Franke, S. I., Henriques, J. A. y Fenech, M. (2012). Iron and genoma stability: An Update. Mutation Research, 733, 92-99. doi: 10.1016/j.mrfmmm.2012.02.001

»Selhub, J., Bagley, L. C., Miller, J. y Rosenberg, I. H. (2000). B vitamins, homocysteine and neurocognitive function in the elderly. American Jorunal of Clinical Nutrition, 71(2), 614S620S. doi: 10.1093/ajcn/71.2.614S

» Sharif, R. P., Thomas, P., Zalewski, R. D., Graham, M. y Fenech, M. (2011). The effect of zinc sulphate and zinc carnosine on genome stability and cytotoxicity in the WIL2- NS human lymphoblastoid cell line, Mutation Research, 720, 22-33. doi: 10.1016/j.mrgentox.2010.12.004

»Sharif, R., Thomas, P., Zalewskib, P. y Fenech M. (2012). The role of zinc in genomic stability. Mutation Research, 733, 111-121. doi: 10.1016/j.mrfmmm.2011.08.009

» Sociedad Argentina de Pediatría. Comité de Crecimiento y Desarrollo (2001). Guías para la evaluación del crecimiento, 2.da edición. Buenos Aires: Sociedad Argentina de Pediatría (SAP).

» Tee, E. S., Kandiah, M., Awin, N., Chong, S. M., Satgunasingam, N., Kamarudin, L., Milani, S., Dugdale, A. E. y Viteri, F. E. (1999). School administered weekly iron-folate supplements improve hemoglobin and ferritin concentrations in Malaysian adolescent girls. American Journal of Clinical Nutrition, 69, 1249-1256. doi: 10.1093/ajcn/69.6.1249

» Terreros, M. C., De Luca, J. C. y Dulout, F. N. (1993). The effect of hypoproteic diet and ethanol consumption on the yield of chromosomal damage detected in bone marrow cells of mice. Journal of Veterinary Medicine Science, 55, 191-194. doi: 10.1292/jvms.55.191

» Thorburn, M. J., Hutchinson, S. y Alleyne, G. A. (1972). Chromosome abnormalities in malnourished children. Lancet, 1, 591. doi: 10.1016/s0140-6736(72)90381-9

» Viteri, F. E., Ali, F. y Tujague, J. (1999). Long-term weekly iron supplementation improves and sustains nonpregnant women's iron status as well or better than currently recommended short-term daily supplementation. Journal of Nutrition, 129, 2013-2020. doi: 10.1093/jn/129.11.2013

»Waterlow, J. C. (1996). Malnutrición proteico-energética. Whashington DC: Publicación Científica no. 555 OPS/OMS.

» Wu, J., Lyons, G. H., Graham, R. D. y Fenech, M. F. (2009). The effect of selenium, as selenomethionine, on genome stability and cytotoxicity inhuman lymphocytes measured using the cytokinesis-block micronucleus cytome assay. Mutagenesis, 24, 225-232. doi: 10.1093/mutage/geno74

» Yan, M., Song, Y., Wong, C. P., Hardin, K. y Ho, E. (2008). Zinc deficiency alters DNA damage response genes in normal human prostate epitelial cells. Journal of Nutrition, 138, 667-673. doi: 10.1093/jn/138.4.667

»Young, M. E. (1996). Desarrollo integral del niño en la primera infancia: desafíos y oportunidades. OPS, OMS, Banco Mundial.

»Zaka-Ur-Rab, Z., Adnan, M., Ahmad, S.M. e Islam, N. (2016). Effect of oral iron on markers of oxidative stress and antioxidant status in children with iron deficiency anaemia. Journal of Clinical Diagnostic Research, 10(10), SC13-SC13. doi: 10.7860/JCDR/2016/23601.8761

»Zalles Cueto, L., Rojas Meneces, J., Rojas Soto, S. y Sejas, E. (2005). Eficacia de la suplementación semanal versus diaria con sulfato ferroso en niños escolares con anemia ferropénica. Gaceta Médica Boliviana, 28(2), 3-8.

» Zhang, S., Hunter, D. J., Hankinson, S. E., Giovannucci, E. L., Rosner, B. A., Colditz, G. A., Speizer, F. E. y Willett, W. C. (1998). A prospective study of folate intake and the risk of breast cancer. JAMA, 281, 1632-1637. doi: 10.1001/jama.281.17.1632 
